\title{
Liouville Theorem for Some Elliptic Equations with Weights and Finite Morse Indices
}

\author{
Qiongli Wu, ${ }^{1,2}$ Liangcai Gan, ${ }^{1}$ and Qingfeng Fan $^{3}$ \\ ${ }^{1}$ Electronic Information School, Wuhan University, Hubei 430071, China \\ ${ }^{2}$ Wuhan Institute of Physics and Mathematics, Chinese Academy of Sciences, Hubei 430071, China \\ ${ }^{3}$ Université de Versailles Saint-Quentin, Laboratoire Données et Algorithmes pour une Ville Intelligente et Durable (DAVID), \\ 78035 Versailles, France
}

Correspondence should be addressed to Qiongli Wu; qiongli.wu@gmail.com

Received 11 December 2015; Revised 20 April 2016; Accepted 21 April 2016

Academic Editor: Simone Secchi

Copyright (C) 2016 Qiongli Wu et al. This is an open access article distributed under the Creative Commons Attribution License, which permits unrestricted use, distribution, and reproduction in any medium, provided the original work is properly cited.

We establish the nonexistence of solution for the following nonlinear elliptic problem with weights: $-\Delta u=\left(1+|x|^{\alpha}\right)|u|^{p-1} u$ in $\mathbb{R}^{N}$, where $\alpha$ is a positive parameter. Suppose that $1<p<(N+2) /(N-2), \alpha>(N-2)(p+1) / 2-N$ for $N \geq 3$ or $p>1, \alpha>-2$ for $N=2$; we will show that this equation does not possess nontrivial bounded solution with finite Morse index.

\section{Introduction}

Liouville theorems are very important in proving a priori bound of solutions for elliptic equations. As far as we know, the most powerful tool for proving the a priori bound is the blow-up method. During the blow-up process, we first suppose that the solutions are unbounded; then we can scale the sequence of solutions. Finally, we get a nontrivial solution for the limit equation. On the other hand, if we can prove that the limit equation does not possess a nontrivial solution, then we get a contradiction. So the solutions must be bounded. From the above statements, it is easy to see that the most important ingredient in proving the a priori bound is the nonexistence result for the limit equation. These kinds of nonexistence results are usually called Liouville type theorems.

For elliptic equations, the first Liouville theorem was proved by Gidas and Spruck in [1], in which the authors proved that the following equation

$$
-\Delta u=u^{p} \quad \text { in } \mathbb{R}^{N}
$$

does not possess positive solutions provided $0<p<(N+$ $2) /(N-2)$. Moreover, it was also proved that the exponent
$(N+2) /(N-2)$ is optimal in the sense that problem (1) indeed possesses a positive solution for $p \geq(N+2) /(N-2)$. So the exponent $(N+2) /(N-2)$ is usually called the critical exponent for problem (1). However, this Liouville theorem is not sufficient for proving the a priori bound since the solutions may blow up on the boundary of the domain. In order to overcome this difficulty, the authors studied the limit equation in the half space

$$
\begin{array}{rr}
-\Delta u=u^{p} & \text { in } \mathbb{R}_{+}^{N}, \\
u=0 & \text { on } \partial \mathbb{R}_{+}^{N}
\end{array}
$$

in [2]. They proved the above equation also does not possess positive solutions provided $0<p<(N+2) /(N-2)$. The above two Liouville theorems are what we need to prove a priori bound for positive solutions of nonlinear elliptic equations in bounded domain. Later, Chen and Li obtained similar nonexistence results for the above two equations in [3] by using the moving plane method.

At the same time, elliptic equations with weights

$$
-\Delta u=|x|^{\alpha} u^{p} \quad \text { in } \mathbb{R}^{N}
$$


were also widely studied and there are many existence and nonexistence results for problem (3). If $\alpha<0$, we say this problem is a Hardy type equation, while for $\alpha>0$, we say this problem is a Henon type equation. For the Hardy type problem, it can be proved that this problem does not possess positive solution provided $0<p<(N+2 \alpha+2) /(N-2)$ by using the moving plane method as in [3]. However, for the Henon type equation, this proof of the nonexistence result is completely open up to now. The main difference between the two cases lies in that, for $\alpha<0$, the weight $|x|^{\alpha}$ is decreasing in $|x|$, so the moving plane method works. However, for $\alpha>$ 0 , the weight $|x|^{\alpha}$ is increasing in $|x|$, so the moving plane method does not work.

On the other hand, we note that the above-mentioned results only claim that the above equations do not possess positive solution. A natural and more difficult question is whether the above equations possess sign-changing solution. However, this question is also completely open up to now. A partial answer was given in [4] in which the authors assume the solution has finite Morse index; then they proved the nonexistence result for this kind of solution. To prove this result, the author first deduced some integrable conditions on the solution based on finite Morse index; then they use the Pohozaev identity to prove the nonexistence result. After this work, there are many extensions on similar problems. For example, Harrabi et al. extended these results to more general nonlinear problems in $[5,6]$. The corresponding Neumann boundary value problems were studied in [7]; Yu studied the mixed boundary problems, the nonlinear boundary value problem, and the fractional Laplacian equation in [8], [9], and [10], respectively.

In this paper, inspired by the above works, we study another problem, that is, the following elliptic equation with weight:

$$
-\Delta u=\left(1+|x|^{\alpha}\right)|u|^{p-1} u \text { in } \mathbb{R}^{N},
$$

where $\alpha>0$ is a positive parameter. We are mainly concerned with the nonexistence of solution with finite Morse index. Because of the interaction of $|u|^{p-1} u$ and $|x|^{\alpha}|u|^{p-1} u$, in order to prove the nonexistence result, we need to add a new bound for the exponent of the weight. More precisely, we have the following result.

Theorem 1. Suppose that $1<p<(N+2) /(N-2), \alpha>$ $(N-2)(p+1) / 2-N$ for $N \geq 3$ or $p>1, \alpha>-2$ for $N=2$; let $u$ be a bounded solution for problem (4) with $i(u)<\infty$; then $u \equiv 0$, where $i(u)$ is the Morse index of $u$.

The rest of this paper is devoted to the proof of the above theorem. We first deduce some inequality based on finite Morse index; then we derive some integral conditions on this solution. Finally, we use the Pohozaev inequality to prove the above theorem. In the following, we denote by $C$ a positive constant, which may vary from line to line.

\section{Proof of Theorem 1}

In this section, we always assume the conditions in Theorem 1 hold. We establish the nonexistence of finite Morse index solution for problem (4). For this purpose, we first recall the definition of Morse index. Let $u$ be a solution of problem (4); we define

$$
\operatorname{dim}\left\{\varphi \in C_{0}^{\infty}\left(\mathbb{R}^{N}\right) \mid\left\langle I^{\prime \prime}(u) \varphi, \varphi\right\rangle<0\right\}
$$

as the Morse index for $u$, where

$$
\begin{aligned}
I(u)= & \frac{1}{2} \int_{\mathbb{R}^{N}}|\nabla u|^{2} d x \\
& -\frac{1}{p+1} \int_{\mathbb{R}^{N}}\left(1+|x|^{\alpha}\right)|u|^{p+1} d x
\end{aligned}
$$

and hence

$$
\begin{aligned}
\left\langle I^{\prime \prime}(u) \varphi, \varphi\right\rangle= & \int_{\mathbb{R}^{N}}|\nabla \varphi|^{2} d x \\
& -p \int_{\mathbb{R}^{N}}\left(1+|x|^{\alpha}\right)|u|^{p-1} \varphi^{2} d x .
\end{aligned}
$$

Also, for further use, we need to define some cut-off function. Let $s>2 r>0$; we define $0 \leq \phi_{r, s} \leq 1$ as follows:

$$
\phi_{r, s}= \begin{cases}0 & \text { for }|x|<r \text { or }|x|>2 s \\ 1 & \text { for } 2 r \leq|x| \leq s .\end{cases}
$$

Moreover, we assume that $\left|\nabla \phi_{r, s}\right| \leq 2 / r$ for $r<|x|<2 r$ and $\left|\nabla \phi_{r, s}\right| \leq 2 / s$ for $s<|x|<2 s$. In the same spirit of [8-10], we have the following result.

Lemma 2. Let $u$ be a solution of (4) with finite Morse index; then there exists $R_{0}>0$ such that

$$
\left\langle I^{\prime \prime}(u) u \phi_{R_{0}, R}, u \phi_{R_{0}, R}\right\rangle \geq 0
$$

for any $R>2 R_{0}$.

Proof. The proof is the same as [8-10]; we omit it.

The next lemma is the key ingredient in the proof of Theorem 1.

Lemma 3. Let $u$ be a bounded solution of problem (4) with finite Morse index and let $\alpha, p$ satisfy the assumptions in Theorem 1; then one has

$$
\begin{array}{r}
\int_{\mathbb{R}^{N}}|u|^{p+1} d x<\infty, \\
\int_{\mathbb{R}^{N}}|x|^{\alpha}|u|^{p+1} d x<\infty, \\
\int_{\mathbb{R}^{N}}|\nabla u|^{2} d x<\infty .
\end{array}
$$


Proof. We will use the information of finite Morse index of $u$ to prove our result. We first prove that $\int_{\mathbb{R}^{N}}|u|^{p+1} d x<\infty$. By Lemma 2, there exists a positive constant $R_{0}>0$, such that

$$
\left\langle I^{\prime \prime}(u) u \phi_{R_{0}, R}, u \phi_{R_{0}, R}\right\rangle \geq 0
$$

for any $R>2 R_{0}$. Then by the definition of $I(u)$, we conclude that

$$
\begin{gathered}
p \int_{\mathbb{R}^{N}}\left(1+|x|^{\alpha}\right)|u|^{p+1} \phi_{R_{0}, R}^{2} d x \\
\leq \int_{\mathbb{R}^{N}}\left|\nabla\left(u \phi_{R_{0}, R}\right)\right|^{2} d x .
\end{gathered}
$$

A direct calculation shows that the right hand side of (12) equals

$$
\int_{\mathbb{R}^{N}}|\nabla u|^{2} \phi_{R_{0}, R}^{2}+\left|\nabla \phi_{R_{0}, R}\right| u^{2}+2 u \phi_{R_{0}, R} \nabla u \nabla \phi_{R_{0}, R} d x
$$

On the other hand, if we multiply (4) by $u \phi_{R_{0}, R}^{2}$ and integrate by parts, then we have

$$
\begin{gathered}
\int_{\mathbb{R}^{N}}|\nabla u|^{2} \phi_{R_{0}, R}^{2}+2 u \phi_{R_{0}, R} \nabla u \nabla \phi_{R_{0}, R} d x \\
=\int_{\mathbb{R}^{N}}\left(1+|x|^{\alpha}\right)|u|^{p+1} \phi_{R_{0}, R}^{2} d x .
\end{gathered}
$$

Inserting (14) into (12), then we get

$$
\begin{aligned}
& (p-1) \int_{\mathbb{R}^{N}}\left(1+|x|^{\alpha}\right)|u|^{p+1} \phi_{R_{0}, R}^{2} d x \\
& \leq \int_{\mathbb{R}^{N}} u^{2}\left|\nabla \phi_{R_{0}, R}\right|^{2} d x \\
& \leq C_{0}+\frac{C}{R^{2}} \int_{\{R \leq|x| \leq 2 R\} \cap \mathbb{R}^{N}} u^{2} d x .
\end{aligned}
$$

In particular, we have

$$
\begin{aligned}
& (p-1) \int_{\mathbb{R}^{N}}|u|^{p+1} \phi_{R_{0}, R}^{2} d x \\
& \leq C_{0}+\frac{C}{R^{2}} \int_{\{R \leq|x| \leq 2 R\} \cap \mathbb{R}^{N}} u^{2} d x, \\
& (p-1) \int_{\mathbb{R}^{N}}|x|^{\alpha}|u|^{p+1} \phi_{R_{0}, R}^{2} d x \\
& \leq C_{0}+\frac{C}{R^{2}} \int_{\{R \leq|x| \leq 2 R\} \cap \mathbb{R}^{N}} u^{2} d x .
\end{aligned}
$$

If $N=2$, we already have $\int_{\mathbb{R}^{N}}|u|^{p+1} \phi_{R_{0}, R}^{2} d x<\infty$ and $\int_{\mathbb{R}^{N}}|x|^{\alpha}|u|^{p+1} \phi_{R_{0}, R}^{2} d x<\infty$ since the right hand sides of (16) and (17) are bounded by a positive constant independent of $R$. This proves the result for $N=2$. So in the following, we always assume that $N \geq 3$. We deduce from (16) and the Holder inequality that

$$
\begin{aligned}
& (p-1) \int_{\mathbb{R}^{N}}|u|^{p+1} \phi_{R_{0}, R}^{2} d x \\
& \leq C_{0}+\frac{C}{R^{2}} \int_{\{R \leq|x| \leq 2 R\} \cap \mathbb{R}^{N}} u^{2} d x \\
& \leq C_{0}+C\left(\int_{\{R \leq|x| \leq 2 R\} \cap \mathbb{R}^{N}}|u|^{p+1} d x\right)^{2 /(p+1)} \\
& \cdot R^{N((p-1) /(p+1))-2} .
\end{aligned}
$$

Suppose on the contrary that $\int_{\mathbb{R}^{N}}|u|^{p+1} d x$ is infinite; then we have

$$
\begin{gathered}
\int_{B_{R}}|u|^{p+1} d x \leq C\left(\int_{B_{2 R}}|u|^{p+1} d x\right)^{2 /(p+1)} \\
\cdot R^{N((p-1) /(p+1))-2}
\end{gathered}
$$

for some $C>0$ and $R$ large enough. Denote $\mu=N((p-$ $1) /(p+1))-2, \theta=2 /(p+1)$, and $J(R)=\int_{B_{R}}|u|^{p+1} d x$; if we iterate the above inequality $k$ times, then we get

$$
J(R) \leq C R^{\mu \gamma} J\left(2^{k+1} R\right)^{\theta^{k+1}}
$$

with $\gamma=1+\theta+\theta^{2}+\cdots+\theta^{k}$. Since $u$ is bounded, a direct calculation shows that the right hand side of (20) is of order $R^{M}$ with

$$
M=\mu \frac{1-\theta^{k+1}}{1-\theta}+N \theta^{k+1} \longrightarrow \frac{\mu}{1-\theta}
$$

as $k \rightarrow \infty$. In particular, we can choose $k$ large enough, such that $M<0$. Then it follows from (20) that

$$
J(R) \longrightarrow 0
$$

as $R \rightarrow \infty$, which is impossible. So we get $\int_{\mathbb{R}^{N}}|u|^{p+1} d x<\infty$.

Next, we show that $\int_{\mathbb{R}^{N}}|x|^{\alpha}|u|^{p+1} d x<\infty$. By the same spirit as the above, we deduce from (17) that

$$
\begin{aligned}
& (p-1) \int_{\mathbb{R}^{N}}|x|^{\alpha}|u|^{p+1} \phi_{R_{0}, R}^{2} d x \\
& \leq C_{0}+\frac{C}{R^{2}} \int_{\{R \leq|x| \leq 2 R\} \cap \mathbb{R}^{N}} u^{2} d x \\
& \leq C_{0}+C\left(\int_{\{R \leq|x| \leq 2 R\} \cap \mathbb{R}^{N}}|x|^{\alpha}|u|^{p+1} d x\right)^{2 /(p+1)} \\
& \cdot R^{-2 \alpha /(p+1)+N((p-1) /(p+1))-2} .
\end{aligned}
$$


Suppose on the contrary that $\int_{\mathbb{R}^{N}}|x|^{\alpha}|u|^{p+1} d x$ is infinite; then we have

$$
\begin{aligned}
\int_{B_{R}}|x|^{\alpha}|u|^{p+1} d x \leq & C\left(\int_{B_{2 R}}|x|^{\alpha}|u|^{p+1} d x\right)^{2 /(p+1)} \\
& \cdot R^{-2 \alpha /(p+1)+N((p-1) /(p+1))-2}
\end{aligned}
$$

for some $C>0$ and $R$ large enough. Denote $\widetilde{\mu}=-2 \alpha /(p+1)+$ $N((p-1) /(p+1))-2$ and $\widetilde{J}(R)=\int_{B_{R}}|x|^{\alpha}|u|^{p+1} d x$; similarly, if we iterate the above inequality $k$ times, then we get

$$
\widetilde{J}(R) \leq C R^{\tilde{\mu} \gamma} \widetilde{J}\left(2^{k+1} R\right)^{\theta^{k+1}}
$$

with $\gamma=1+\theta+\theta^{2}+\cdots+\theta^{k}$. By the boundedness of $u$, a direct calculation shows that the right hand side of (25) is of order $R^{\widetilde{M}}$ with

$$
\widetilde{M}=\widetilde{\mu} \frac{1-\theta^{k+1}}{1-\theta}+(\alpha+N) \theta^{k+1} \longrightarrow \frac{\widetilde{\mu}}{1-\theta}
$$

as $k \rightarrow \infty$. So we can still choose $k$ large enough, such that $\widetilde{M}<0$. Then it follows from (25) that

$$
J(R) \longrightarrow 0
$$

as $R \rightarrow \infty$, which is a contradiction. So we get that $\int_{\mathbb{R}^{N}}|x|^{\alpha}|u|^{p+1} d x<\infty$.

Finally, we show that $\int_{\mathbb{R}^{N}}|\nabla u|^{2} d x<\infty$. For this purpose, we first choose a cut-off function $0 \leq \varphi \leq 1$ such that $\varphi=1$ for $|x| \leq 1$ and $\varphi=0$ for $|x| \geq 2$. For any $R>0$, we multiply (4) by $u \varphi(x / R)$ and integrate by parts; then we get

$$
\begin{aligned}
\int_{B_{2 R}}|u|^{p+1} \varphi\left(\frac{x}{R}\right) d x= & \int_{B_{2 R}}-\Delta u u \varphi\left(\frac{x}{R}\right) d x \\
= & \int_{B_{2 R}}|\nabla u|^{2} \varphi\left(\frac{x}{R}\right) d x \\
& +\frac{1}{R} \int_{B_{2 R}} u \nabla u \nabla \varphi\left(\frac{x}{R}\right) d x \\
= & \int_{B_{2 R}}|\nabla u|^{2} \varphi\left(\frac{x}{R}\right) d x \\
& -\frac{1}{2 R^{2}} \int_{B_{2 R}} u^{2} \Delta \varphi\left(\frac{x}{R}\right) d x .
\end{aligned}
$$

Since

$$
\begin{aligned}
& \frac{1}{2 R^{2}} \int_{B_{2 R}} u^{2} \Delta \varphi\left(\frac{x}{R}\right) d x \leq \frac{C}{R^{2}} \int_{B_{2 R}} u^{2} d x \\
& \quad \leq C R^{-2+N((p-1) /(p+1))}\left(\int_{B_{2 R}}|u|^{p+1} d x\right)^{2 /(p+1)},
\end{aligned}
$$

we infer from the above two equations that

$$
\begin{aligned}
& \int_{B_{2 R}}|\nabla u|^{2} \varphi\left(\frac{x}{R}\right) d x \\
& \leq C R^{-2+N((p-1) /(p+1))}\left(\int_{B_{2 R}}|u|^{p+1} d x\right)^{2 /(p+1)} \\
& \quad+\int_{B_{2 R}}|u|^{p+1} \varphi\left(\frac{x}{R}\right) .
\end{aligned}
$$

By the assumption $p<(N+2) /(N-2)$ for $N \geq 3$ and the fact that $\int_{\mathbb{R}^{N}}|u|^{p+1} d x<\infty$, we get

$$
\int_{\mathbb{R}^{N}}|\nabla u|^{2} d x<\infty
$$

by letting $R \rightarrow \infty$ in the above equation. This finishes the proof of this lemma.

In order to complete the proof of Theorem 1, we need the following Pohozaev identity for problem (4).

Lemma 4. Suppose that $u$ is a solution of (4); then the following identity holds:

$$
\begin{gathered}
\frac{N-2}{2} \int_{B_{R}}|\nabla u|^{2} d x-\frac{N}{p+1} \int_{B_{R}}|u|^{p+1} d x \\
-\frac{N+\alpha}{p+1} \int_{B_{R}}|x|^{\alpha}|u|^{p+1} d x \\
=\frac{R}{2} \int_{\partial B_{R}}|\nabla u|^{2} d S-R \int_{\partial B_{R}}\left|\frac{\partial u}{\partial \nu}\right|^{2} d S \\
\quad-\frac{R}{p+1} \int_{\partial B_{R}}|u|^{p+1} d S \\
-\frac{R}{p+1} \int_{\partial B_{R}}|x|^{\alpha}|u|^{p+1} d S .
\end{gathered}
$$

Proof. The proof of this lemma is standard; we give the details to keep this paper self-contained.

Multiplying (4) by $\langle x, \nabla u\rangle$ and integrating in $B_{R}$, then we get

$$
-\int_{B_{R}} \Delta u\langle x, \nabla u\rangle=\int_{B_{R}}\left(1+|x|^{\alpha}\right)|u|^{p-1} u\langle x, \nabla u\rangle .
$$

The left hand side of (33) equals

$$
\begin{aligned}
-\int_{B_{R}} \Delta u\langle x, \nabla u\rangle= & \int_{B_{R}} \nabla u \nabla\langle x, \nabla u\rangle d x \\
& -\int_{\partial B_{R}} \frac{\partial u}{\partial \nu}\langle x, \nabla u\rangle d S \\
= & \int_{B_{R}}|\nabla u|^{2} d x \\
& +\frac{1}{2} \int_{B_{R}}\left\langle x, \nabla\left(|\nabla u|^{2}\right)\right\rangle d x
\end{aligned}
$$




$$
\begin{aligned}
& -\int_{\partial B_{R}} \frac{\partial u}{\partial \nu}\langle x, \nabla u\rangle d S \\
= & -\frac{N-2}{2} \int_{B_{R}}|\nabla u|^{2} d x \\
& +\frac{1}{2} \int_{\partial B_{R}}\langle x, v\rangle|\nabla u|^{2} d S \\
& -\int_{\partial B_{R}} \frac{\partial u}{\partial \nu}\langle x, \nabla u\rangle d S \\
= & -\frac{N-2}{2} \int_{B_{R}}|\nabla u|^{2} d x \\
& +\frac{R}{2} \int_{\partial B_{R}}|\nabla u|^{2} d S \\
& -R \int_{\partial B_{R}}\left|\frac{\partial u}{\partial \nu}\right|^{2} d S,
\end{aligned}
$$

while the right hand side of (33) equals

$$
\begin{aligned}
\int_{B_{R}}(1 & \left.+|x|^{\alpha}\right)|u|^{p-1} u\langle x, \nabla u\rangle d x \\
= & \frac{1}{p+1} \int_{B_{R}}\left\langle x, \nabla|u|^{p+1}\right\rangle d x \\
& +\frac{1}{p+1} \int_{B_{R}}\left\langle|x|^{\alpha} x, \nabla|u|^{p+1}\right\rangle d x \\
= & -\frac{N}{p+1} \int_{B_{R}}|u|^{p+1} d x \\
& +\frac{1}{p+1} \int_{\partial B_{R}}\langle x, v\rangle|u|^{p+1} d S \\
& -\frac{N+\alpha}{p+1} \int_{B_{R}}|x|^{\alpha}|u|^{p+1} d x \\
& +\frac{1}{p+1} \int_{\partial B_{R}}\langle x, \nu\rangle|x|^{\alpha}|u|^{p+1} d S \\
= & -\frac{N}{p+1} \int_{B_{R}}|u|^{p+1} d x+\frac{R}{p+1} \int_{\partial B_{R}}|u|^{p+1} d S \\
& -\frac{N+\alpha}{p+1} \int_{B_{R}}|x|^{\alpha}|u|^{p+1} d x \\
& +\frac{R}{p+1} \int_{\partial B_{R}}|x|^{\alpha}|u|^{p+1} d S .
\end{aligned}
$$

Combining the above two equations together, then we get the above local Pohozaev identity for problem (4).

With the above preparations, we can prove Theorem 1 now.
Proof of Theorem 1. First, since $\int_{\mathbb{R}^{N}}|u|^{p+1} d x<\infty$, $\int_{\mathbb{R}^{N}}|x|^{\alpha}|u|^{p+1} d x<\infty$, and $\int_{\mathbb{R}^{N}}|\nabla u|^{2} d x<\infty$ by Lemma 3, then there exists a sequence $R_{n} \rightarrow \infty$ such that

$$
\begin{array}{r}
\frac{R_{n}}{2} \int_{\partial B_{R_{n}}}|\nabla u|^{2} d S \longrightarrow 0, \\
R_{n} \int_{\partial B_{R_{n}}}\left|\frac{\partial u}{\partial \nu}\right|^{2} d S \longrightarrow 0, \\
\frac{R_{n}}{p+1} \int_{\partial B_{R_{n}}}|u|^{p+1} d S \longrightarrow 0, \\
\frac{R_{n}}{p+1} \int_{\partial B_{R_{n}}}|x|^{\alpha}|u|^{p+1} d S \longrightarrow 0
\end{array}
$$

as $n \rightarrow \infty$. Let $R=R_{n}$ in the local Pohozaev identity and let $n \rightarrow \infty$; then we get

$$
\begin{aligned}
\frac{N-2}{2} \int_{\mathbb{R}^{N}}|\nabla u|^{2} d x= & \frac{N}{p+1} \int_{\mathbb{R}^{N}}|u|^{p+1} d x \\
& +\frac{N+\alpha}{p+1} \int_{\mathbb{R}^{N}}|x|^{\alpha}|u|^{p+1} d x .
\end{aligned}
$$

Next, multiplying (4) by $u$ and integrating by parts, then we get

$$
\int_{\mathbb{R}^{N}}|\nabla u|^{2} d x=\int_{\mathbb{R}^{N}}|u|^{p+1} d x+\int_{\mathbb{R}^{N}}|x|^{\alpha}|u|^{p+1} d x .
$$

We infer from the above two identities that

$$
\begin{aligned}
& \left(\frac{N-2}{2}-\frac{N}{p+1}\right) \int_{\mathbb{R}^{N}}|u|^{p+1} d x \\
& \quad=\left(\frac{N+\alpha}{p+1}-\frac{N-2}{2}\right) \int_{\mathbb{R}^{N}}|x|^{\alpha}|u|^{p+1} d x .
\end{aligned}
$$

By the assumptions on the exponents $p$ and $\alpha$ in Theorem 1 , we have

$$
\begin{aligned}
& \frac{N-2}{2}-\frac{N}{p+1}<0, \\
& \frac{N+\alpha}{p+1}-\frac{N-2}{2}>0 .
\end{aligned}
$$

For (39) to hold, the only possibility is

$$
\int_{\mathbb{R}^{N}}|u|^{p+1} d x=0
$$

which finally implies $u \equiv 0$. 


\section{Competing Interests}

The authors declare that there are no competing interests regarding the publication of this paper.

\section{References}

[1] B. Gidas and J. Spruck, "Global and local behavior of positive solutions of nonlinear elliptic equations," Communications on Pure and Applied Mathematics, vol. 34, no. 4, pp. 525-598, 1981.

[2] B. Gidas and J. Spruck, "A priori bounds for positive solutions of nonlinear elliptic equations," Communications in Partial Differential Equations, vol. 6, no. 8, pp. 883-901, 1981.

[3] W. X. Chen and C. Li, "Classification of solutions of some nonlinear elliptic equations," Duke Mathematical Journal, vol. 63, no. 3, pp. 615-622, 1991.

[4] A. Bahri and P.-L. Lions, "Solutions of superlinear elliptic equations and their morse indices," Communications on Pure and Applied Mathematics, vol. 45, no. 9, pp. 1205-1215, 1992.

[5] A. Harrabi, S. Rebhi, and S. Selmi, "Solutions of superlinear equations and their Morse indices I," Duke Mathematical Journal, vol. 94, pp. 141-157, 1998.

[6] A. Harrabi, S. Rebhi, and A. Selmi, "Solutions of superlinear elliptic equations and their Morse indices, II," Duke Mathematical Journal, vol. 94, no. 1, pp. 159-179, 1998.

[7] A. Harrabi, M. O. Ahmedou, S. Rebhi, and A. Selmi, "A priori estimates for superlinear and subcritical elliptic equations: the Neumann boundary condition case," Manuscripta Mathematica, vol. 137, no. 3-4, pp. 525-544, 2012.

[8] X. Yu, "Solutions of the mixed boundary problem and their Morse indices," Nonlinear Analysis: Theory, Methods \& Applications, vol. 96, pp. 146-153, 2014.

[9] X. Yu, "Liouville theorem for elliptic equations with nonlinear boundary value conditions and finite Morse indices," Journal of Mathematical Analysis and Applications, vol. 421, no. 1, pp. 436443, 2015.

[10] X. Yu, "Solutions of fractional Laplacian equations and their Morse indices," Journal of Differential Equations, vol. 260, no. 1, pp. 860-871, 2016. 


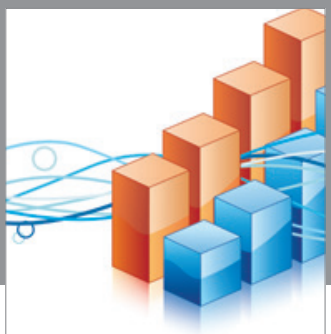

Advances in

Operations Research

vatem alat4

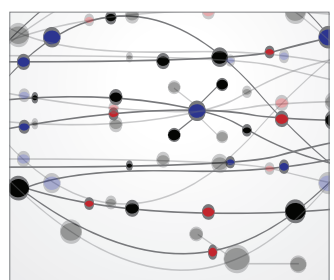

\section{The Scientific} World Journal
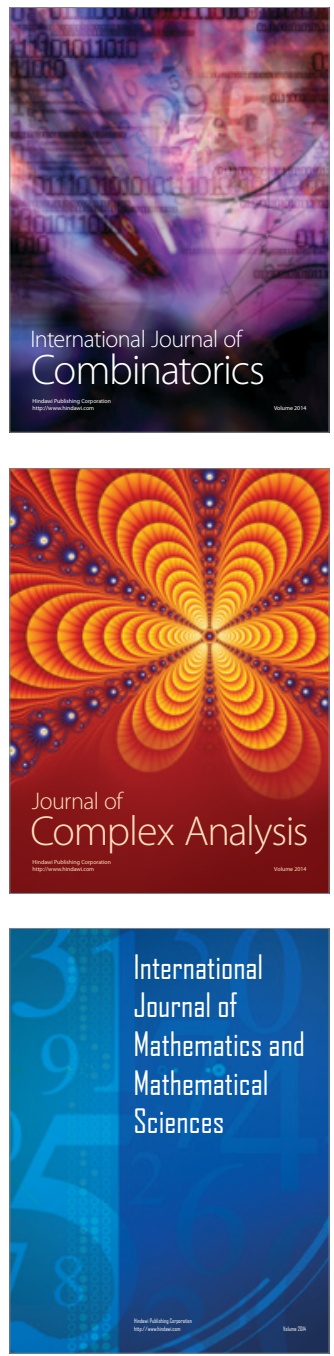
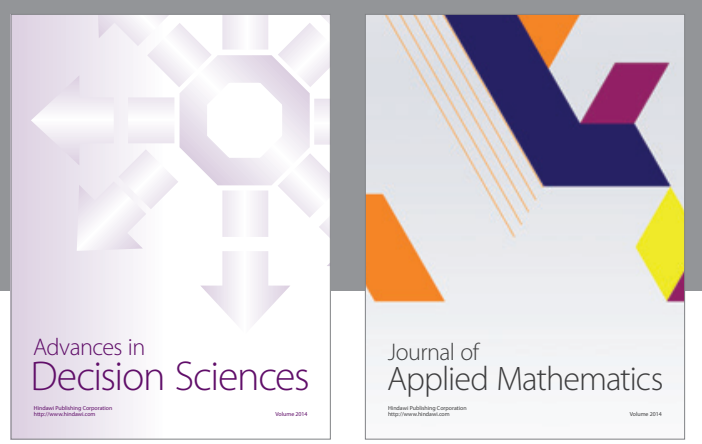

Algebra

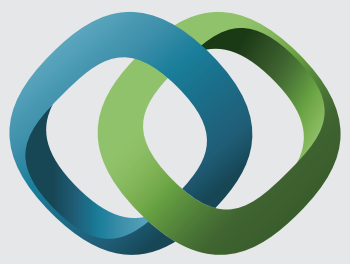

\section{Hindawi}

Submit your manuscripts at

http://www.hindawi.com
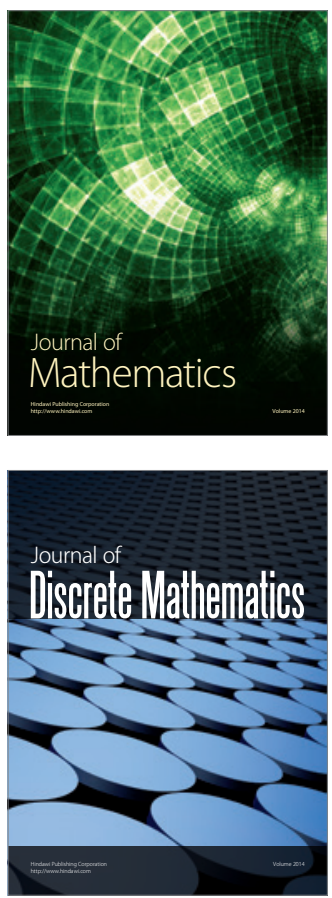

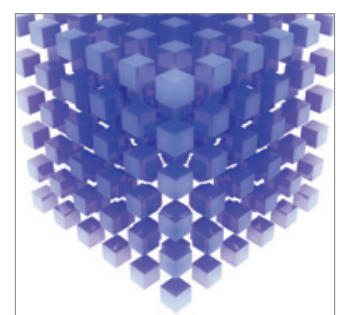

Mathematical Problems in Engineering
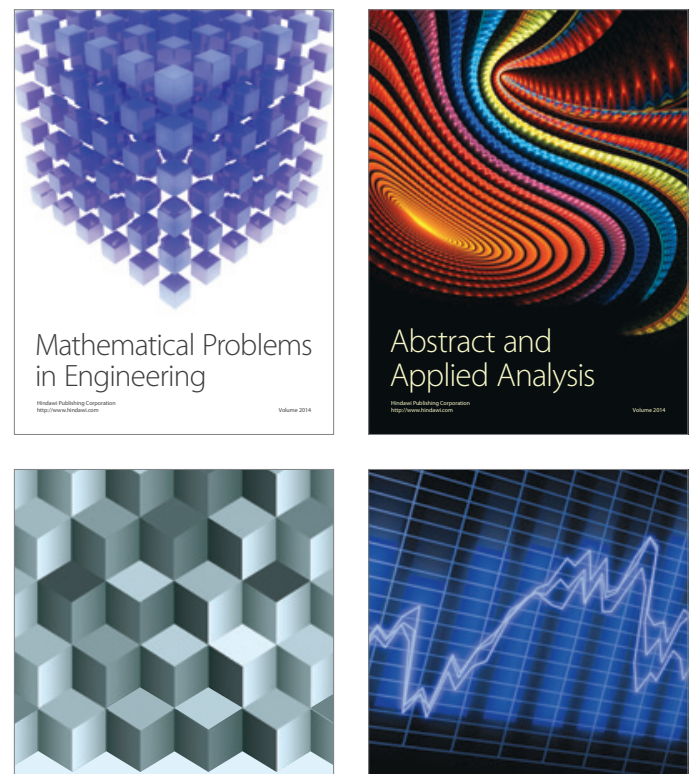

Journal of

Function Spaces

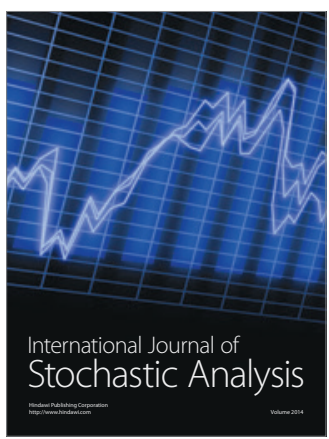

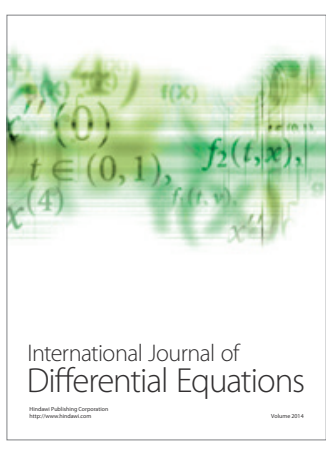
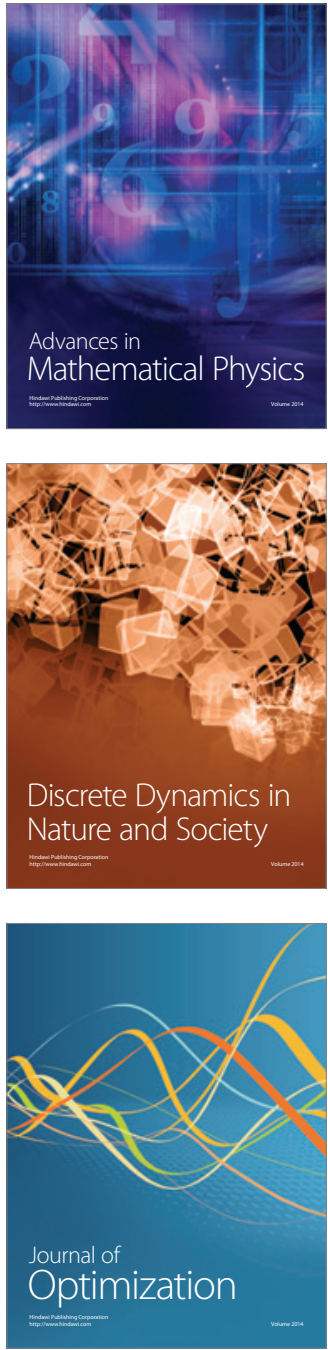